\title{
Cross-linker-Modulated Nanogel Flexibility Correlates with Tunable Targeting to a Sterically Impeded Endothelial Marker
}

\author{
Jacob Wheatley Myerson¹, Olivia McPherson*2, Kelsey G. DeFrates ${ }^{2}$, Jenna H. \\ Towslee $^{2}$, Oscar A. Marcos-Contreras ${ }^{1}$, Vladimir V. Shuvaev¹, Bruce Braender ${ }^{2}$, Russell \\ J. Composto ${ }^{3}$, Vladimir R. Muzykantov ${ }^{1}$, David M. Eckmann ${ }^{2,3}$
}

\section{${ }^{*}$ These authors contributed equally}

${ }^{1}$ Department of Pharmacology, Perelman School of Medicine, University of

Pennsylvania, Philadelphia, PA 19104

${ }^{2}$ Department of Anesthesiology and Critical Care, Perelman School of Medicine, University of Pennsylvania, Philadelphia, PA 19104

${ }^{3}$ Department of Bioengineering, School of Engineering and Applied Sciences, University of Pennsylvania, Philadelphia, PA 19104

\section{Address for Correspondence:}

Vladimir R. Muzykantov, MD, PhD

10-105 Smilow Center for Translational Research

3400 Civic Center Blvd

Philadelphia, PA 19104-5158

muzykant@pennmedicine.upenn.edu 


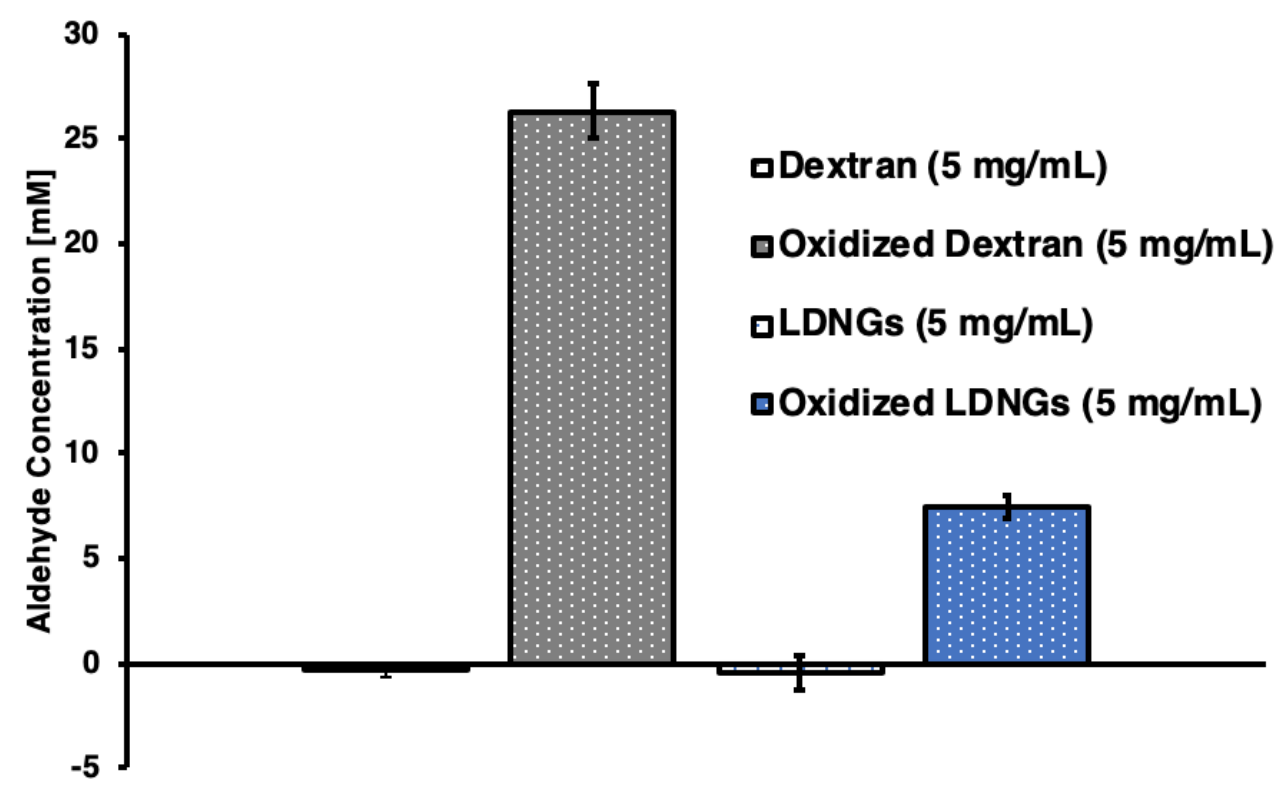

\section{Supplementary Figure 1}

Quantification of aldehyde induction by oxidation of dextran and lysozyme-dextran nanogels. Chromogenic dye assay was used to quantify aldehydes on dextran or NGs after periodate oxidation. Colorimetric assessment was also applied to dextran and NGs prior to oxidation, providing a negative control on the assays for oxidized dextran and oxidized NGs. Aldehyde concentrations of $-0.32 \pm 0.40 \mathrm{mM}$ and $26.34 \pm 1.24 \mathrm{mM}$ were measured for $5 \mathrm{mg} / \mathrm{mL}$ dextran and $5 \mathrm{mg} / \mathrm{mL}$ oxidized dextran, respectively. Aldehyde concentrations of $-0.44 \pm 0.84 \mathrm{mM}$ and $7.43 \pm 1.95 \mathrm{mM}$ were measured for $5 \mathrm{mg} / \mathrm{mL}$ $\mathrm{NGs}$ and $5 \mathrm{mg} / \mathrm{mL}$ oxidized $\mathrm{NGs}$, respectively. 


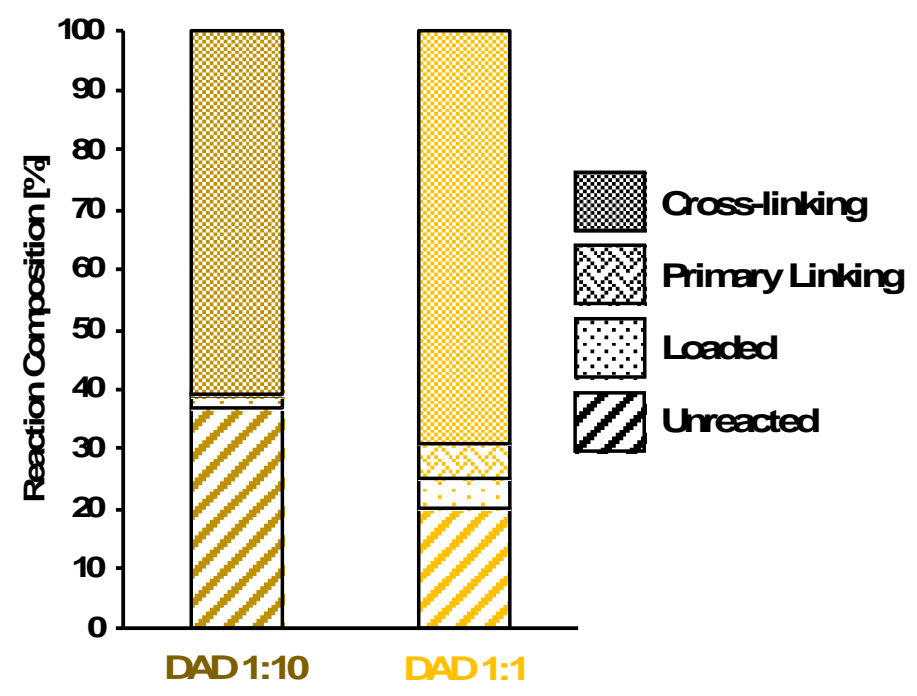

\section{Supplementary Figure 2}

Effect of 1,12-diaminododecane (DAD) concentration on diamine cross-linking of lysozyme-dextran nanogels (NGs). At 1:4 cross-linker mass:NG mass, $69.12 \%$ of the introduced DAD incorporated in cross-linking, 5.81\% formed primary linkages (single bonds without completing a cross-linking reaction), and $4.98 \%$ loaded into the particles without forming bonds. At 1:40 cross-linker mass:NG mass, $60.86 \%$ of the DAD participated in cross-linking, $0.24 \%$ developed primary linkages, and $2.03 \%$ loaded into the particles. At the lesser mass of introduced DAD, 36.87\% of the cross-linker remained unassociated with particles, compared to $20.09 \%$ for the larger DAD:NG ratio. 

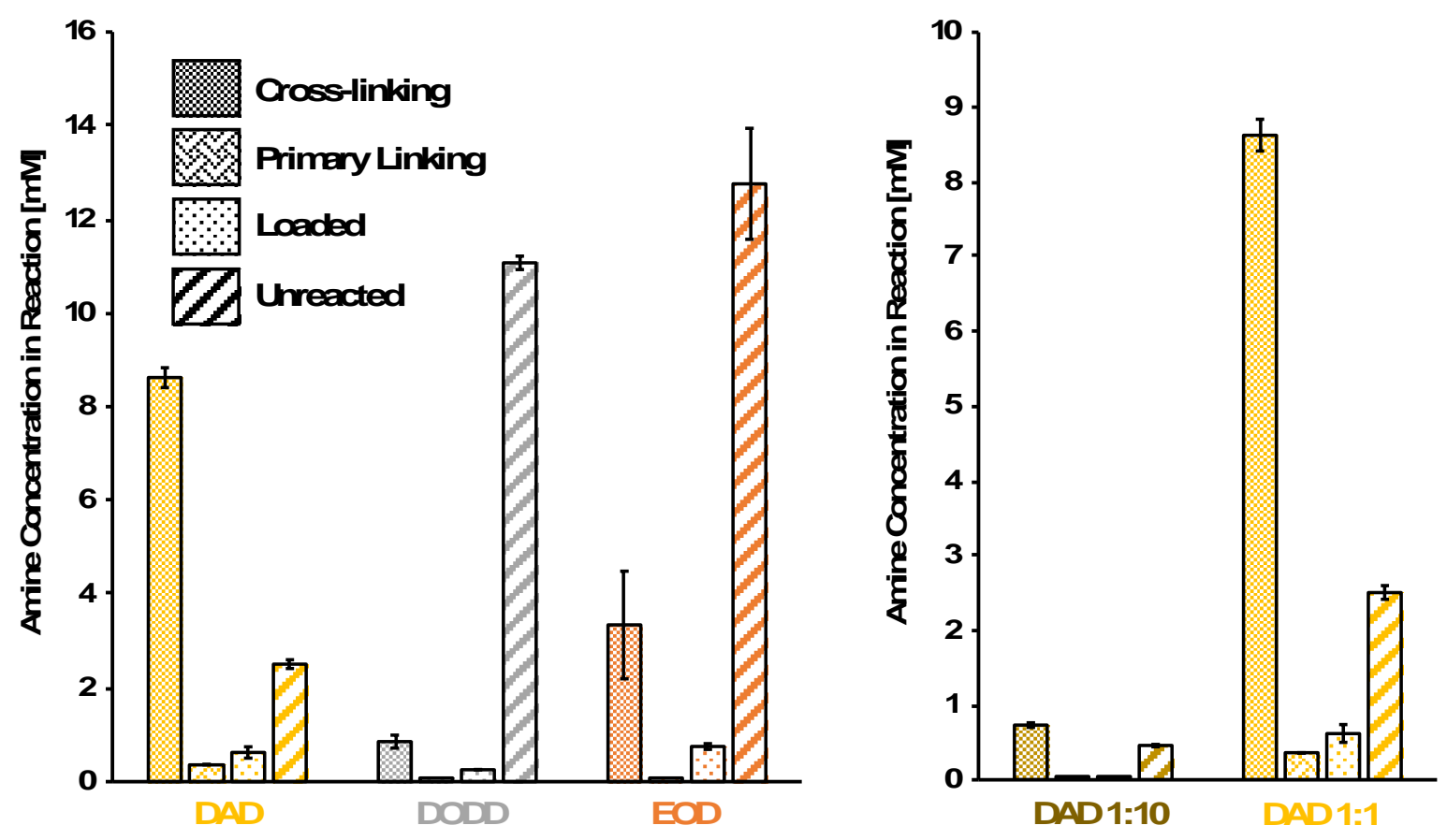

\section{Supplementary Figure 3}

Disposition of 1,12-diaminododecane (DAD, yellow), 4,9-dioxa-1,12-dodecanediamine (DODD, gray), or 2,2'-(ethylenedioxy)bis(ethylamine) (EOD, orange) cross-linker in reactions with NGs. Data represent an alternative analysis of data in Figure $1 \mathrm{E}$ and Supplementary Figure 1, representing variability of the measurements and converting reaction composition to concentration of amines in the aldehyde-amine reactions. Amines incorporated in NG cross-linking accounted for a concentration of $8.62 \pm 0.21$ $\mathrm{mM}$ for DAD, $0.85 \pm 0.14 \mathrm{mM}$ for DODD, and $3.34 \pm 1.15 \mathrm{mM}$ for EOD. Primary linking (diamines forming a single aldehyde-amine bond) amounted to a concentration of 0.36 $\pm 0.002 \mathrm{mM}$ for DAD, $0.03 \pm 0.002 \mathrm{mM}$ for DODD, and $0.009 \pm 0.005 \mathrm{mM}$ for EOD. Cross-linker loading in NGs accounted for a $0.62 \pm 0.12 \mathrm{mM}$ amine concentration for $\mathrm{DAD}, 0.25 \pm 0.005 \mathrm{mM}$ for DODD, and $0.75 \pm 0.06 \mathrm{mM}$ for EOD. A ten-fold reduction in the concentration of DAD in the cross-linking reactions yielded $0.73 \pm 0.03 \mathrm{mM}$ amines engaged in cross-linking, $0.01 \pm 0.005 \mathrm{mM}$ amines engaged in primary linking, and 0.03 $\pm 0.01 \mathrm{mM}$ amines loaded in NGs. 

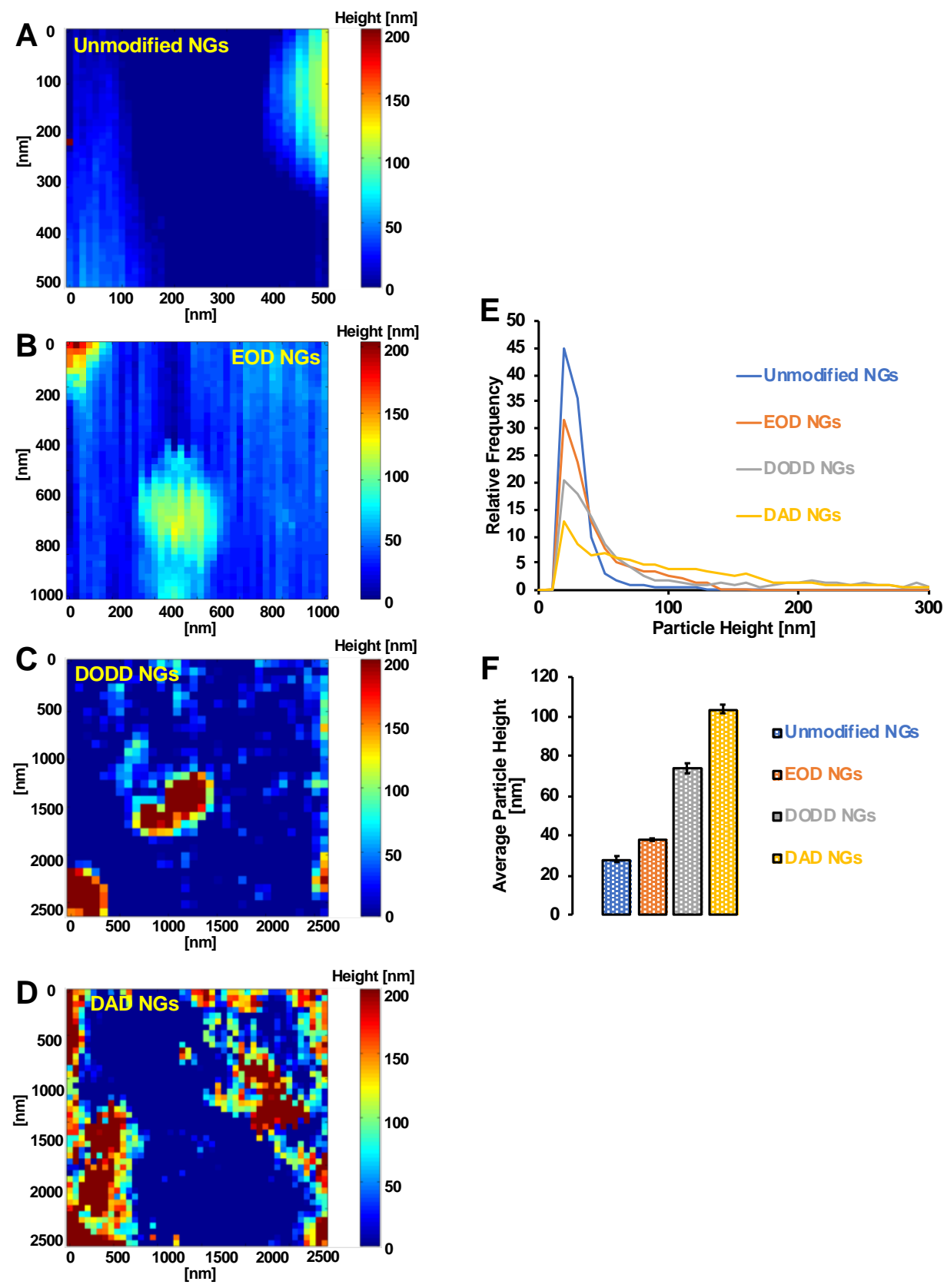

\section{Supplementary Figure 4}

Effect of cross-linking on surface-adhered NG height in AFM micrographs. (a), (b), (c), and (d) depict example AFM micrographs for unmodified NGs, EOD-cross-linked NGs, DODD-cross-linked NGs, and DAD-cross-linked NGs, respectively. After thresholding micrographs above a $20 \mathrm{~nm}$ cutoff height to distinguish nanogels from substrate, pixelby-pixel height averages for each type of NG were determined to produce the histograms depicted in (e) and the average heights depicted in (f). For unmodified NGs $(n=10$ micrographs), we determine an average particle height of $28.06 \pm 1.49 \mathrm{~nm}$. For EOD-cross-linked NGs ( $n=5$ micrographs), we determine an average particle height of $37.88 \pm .66 \mathrm{~nm}$. For DODD-cross-linked NGs ( $\mathrm{n}=4$ micrographs), we determine an average particle height of $73.88 \pm 2.56 \mathrm{~nm}$. For DAD-cross-linked NGs $(\mathrm{n}=4)$, we determine an average particle height of $103.82 \pm 2.19 \mathrm{~nm}$. 


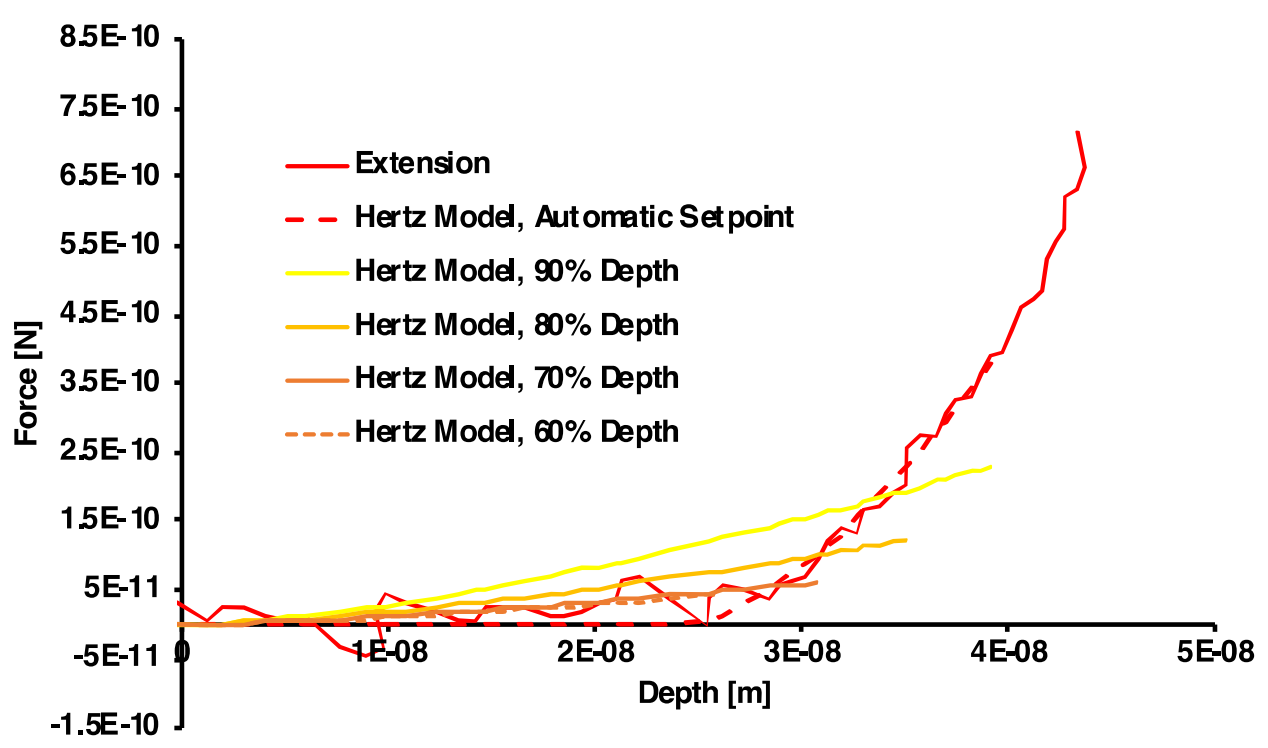

\section{Supplementary Figure 5}

Example force-indentation curve for EOD-cross-linked NGs depicting different Hertzian fitting strategies used to analyze the probe extension curve. Resistance encountered by the probe during extension is depicted in red, with fitting via automatic setpoint and full depth (as determined by threshold resistance of $400 \mathrm{pN}$ ) noted with dashed red line. Yellow, orange, dark orange, and dashed orange curves indicate Hertzian fitting of the extension curve with a setpoint at the NG surface and data terminating at $90 \%, 80 \%$, $70 \%$, and $60 \%$ of the NG depth, respectively. Greater indentation depth and deeper indentation setpoints resulted in larger modeled Young's moduli, indicated by slope of the fitting curves. 

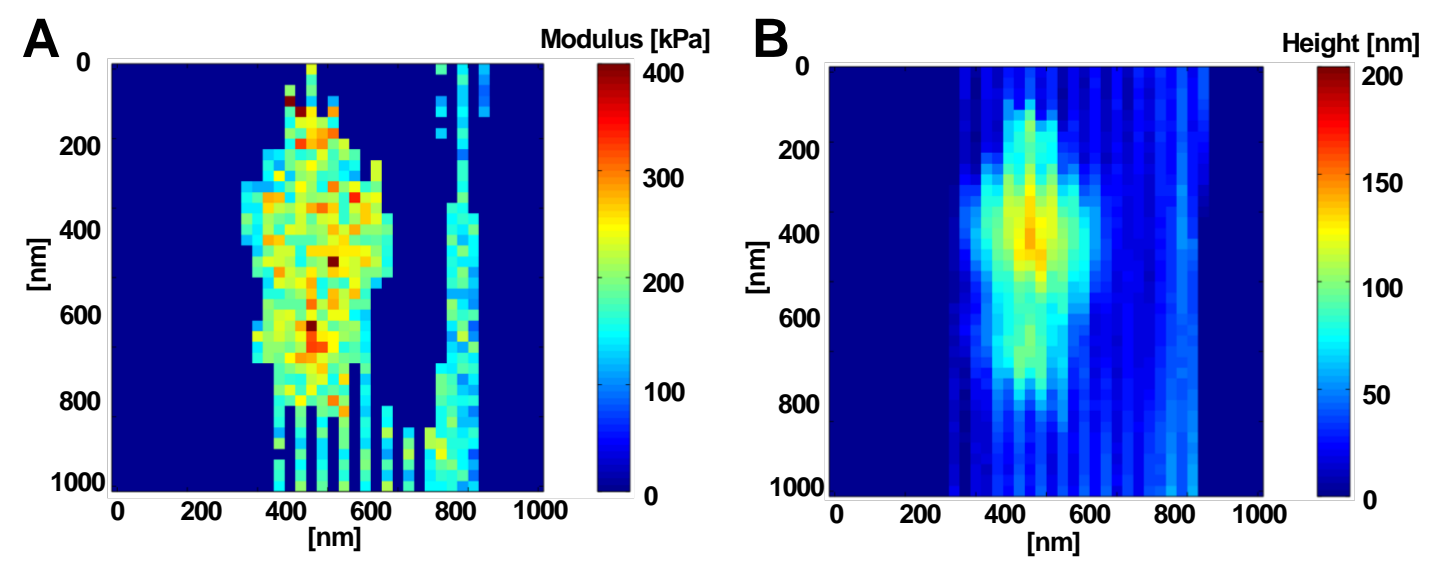

\section{Supplementary Figure 6}

Maps of automatic setpoint/full particle Hertzian Young's moduli for EOD-cross-linked NGs (a) and EOD-cross-linked NG height for the same particles (b). Depicted maps correspond to the particles analyzed in histograms and Young's modulus maps in figure $3 a, 3 b$, and $3 c$ in the main text. 


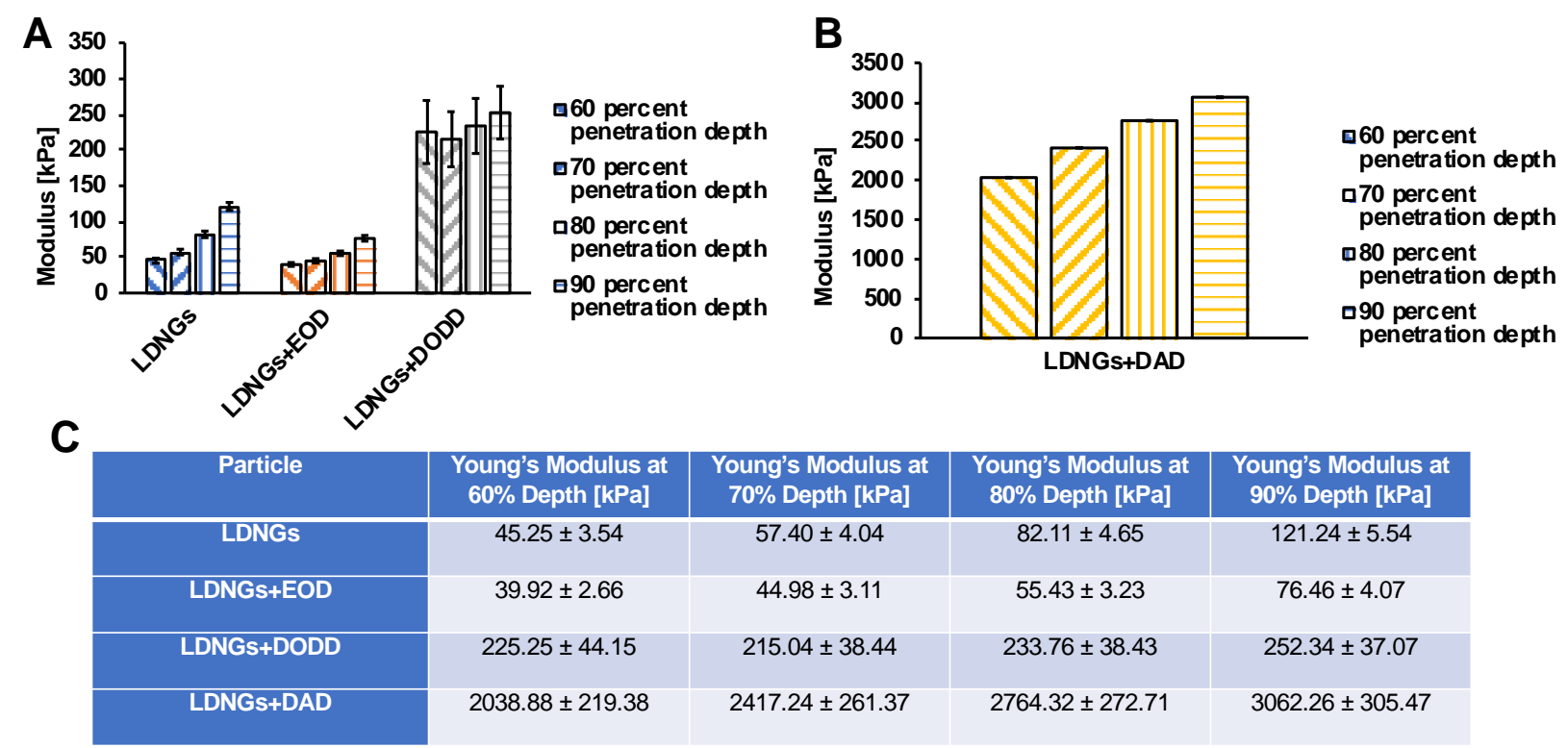

\section{Supplementary Figure 7}

Variation of NG modulus with respect to depth into NGs, recapitulating data presented in figure $3 e$ in the main text with scales altered for clarity. (a) and (b) depict average Young's moduli for NGs at $60 \%, 70 \%, 80 \%$, and $90 \%$ depth of the dextran shell. Unmodified NGs (blue bars, $n=10$ micrographs), EOD-cross-linked NGs (orange bars, $\mathrm{n}=5$ micrographs), and DODD-cross-linked NGs (gray bars, $\mathrm{n}=4$ micrographs) are described in (a) and DAD-cross-linked NGs (yellow bars, $n=4$ micrographs) are described in (b). Moduli for all NG types as a function of depth are further noted in tabular form in (c). For unmodified NGs, EOD-cross-linked NGs, and DAD-cross-linked NGs, modeled Young's modulus increased with indentation depth. For DODD-crosslinked NGs, there were no significant differences in Young's modulus with respect to indentation depth. 

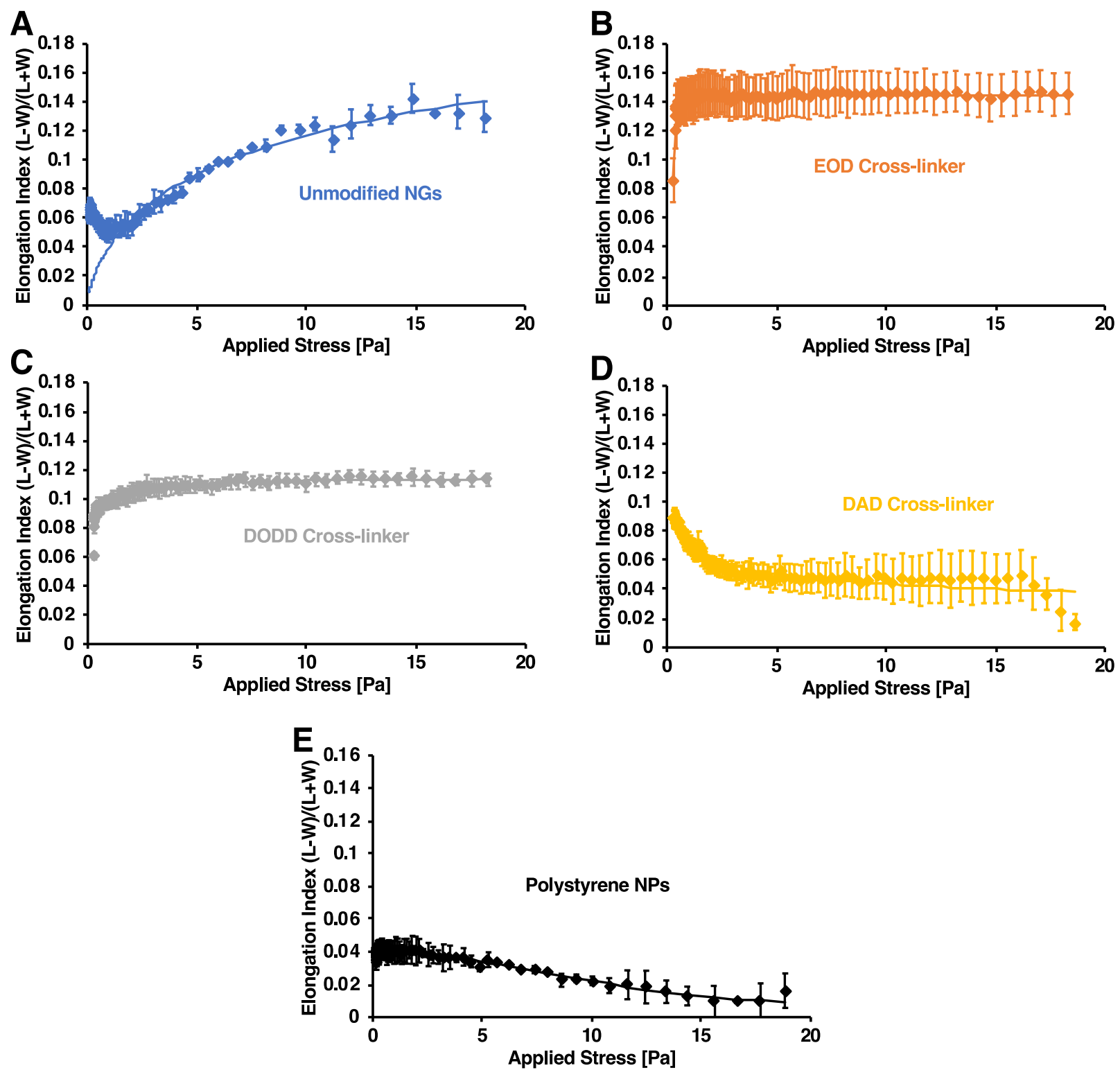

\section{Supplementary Figure 8}

Ektacytometry data indicating deformation of NGs or polystyrene NPs in response to shear in flowing viscous (5.5\% poly(vinyl)pyrrolidone) suspensions. NG suspensions were subjected to varying shear and elongation of the elliptical diffraction patterns generated by flowing NG suspensions was determined for each value of applied shear. Increasing elongation with respect to shear was noted for unmodified NGs (blue points, $a, n=3$ ), EOD-cross-linked NGs (orange points, b, $n=3$ ), and DODD-cross-linked NGs (gray points, $c, n=3$ ). For DAD-cross-linked NGs (yellow points, $d, n=3$ ) and polystyrene NPs (black points, e, $n=3$ ), diffraction patterns did not elongate with increasing shear. 


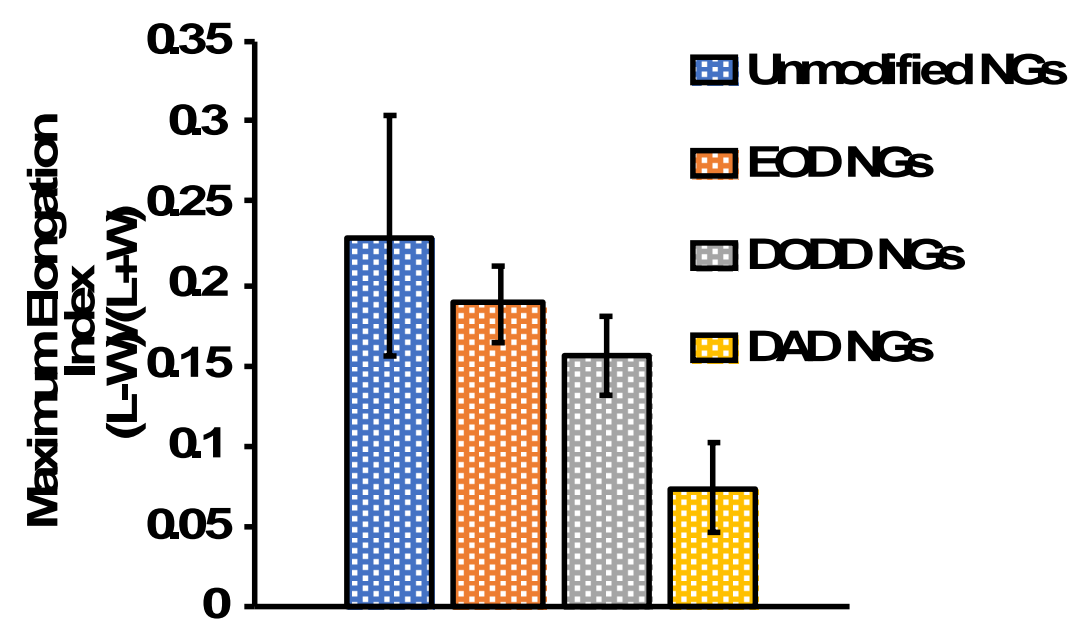

\section{Supplementary Figure 9}

Data in supplementary figure 6 were fitted according to the Streekstra-Bronkhorst model developed for modeling of cell elongation in flow:

$$
E I=\left\{\left(E I_{\text {max }}-E I_{\text {min }}\right) * \frac{\left(\frac{s}{s S_{\text {half }}}\right)^{m}}{1+\left(\frac{s}{s s_{\text {half }}}\right)^{m}}\right\}+E I_{\text {min }},
$$

where $S$ is the applied shear, $E I$ is the elongation index of the diffraction pattern (defined as $(\mathrm{L}-\mathrm{W}) /(\mathrm{L}+\mathrm{W})$, where $\mathrm{L}$ is the length of the diffraction pattern along the flow direction and $\mathrm{W}$ is the length of the diffraction pattern orthogonal to the flow direction), EI $I_{\max }$ is the fitted elongation index at infinite stress, EImin is the fitted elongation index at zero stress, SShalf is the applied stress at which the elongation index reaches half the projected maximum elongation, and $\mathrm{m}$ is a fitting parameter accounting for variations in slope of the EI vs. S curve. Averages of three Streekstra-Bronkhorst fits, depicted as curves in Supplementary Figure 6, determined predicted maximum elongation indices for unmodified NGs and each type of cross-linked NGs. Unmodified NGs, EOD-crosslinked NGs, and DODD-cross-linked NGs had indistinguishable maximum elongation indices. DAD-cross-linked NGs had a significantly lesser maximum elongation index. 
A NG addition to QCM substrate

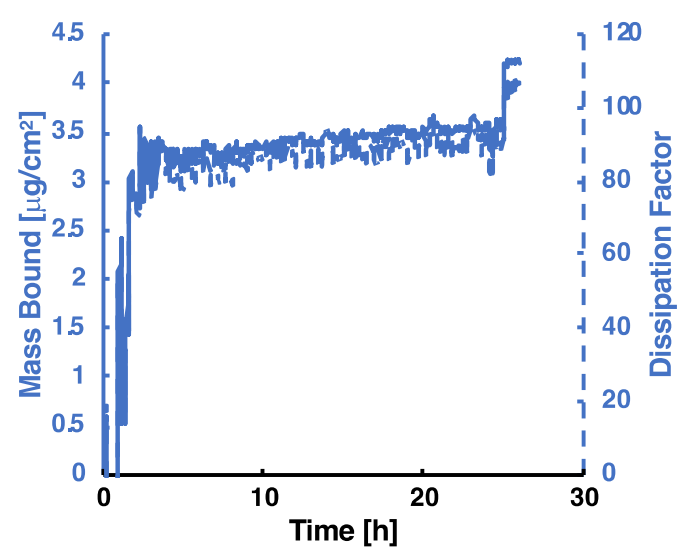

B DAD addition to bound NGs
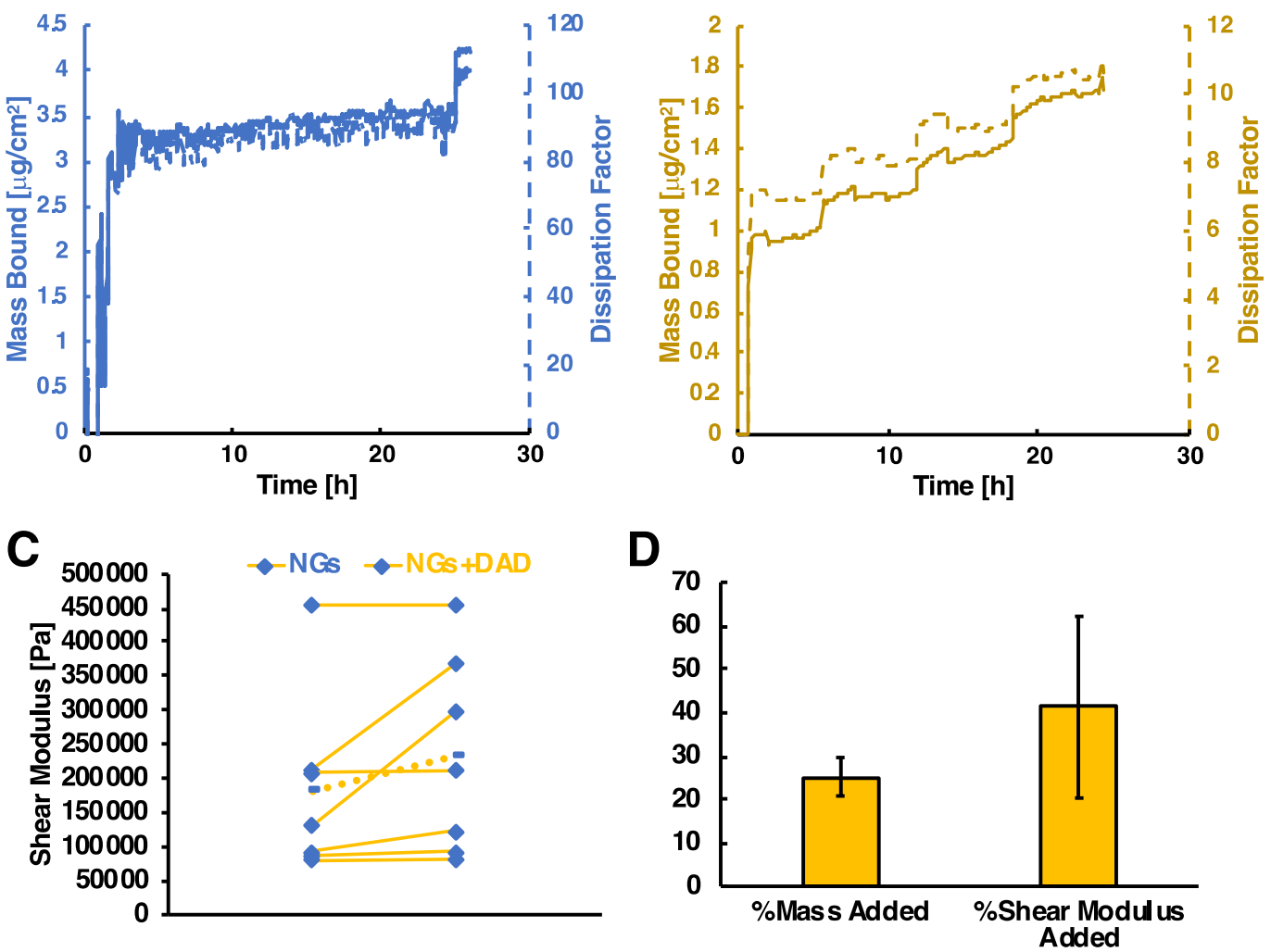

\section{Supplementary Figure 10}

Quartz crystal microbalance (QCM) analysis of DAD cross-linking of NGs. Oxidized aldehyde-presenting NGs were exposed at $5 \mathrm{mg} / \mathrm{mL}$ and $0.007 \mathrm{~mL} / \mathrm{min}$ flow to aminepresenting (3 aminopropyl)triethoxysilane (APTES) QCM surfaces, permitting accumulation of NGs on the complementary surface over 24 hours (example mass accumulation and dissipation factor traces in (a)). After rinsing unbound NGs, DAD was exposed to the QCM surface-grafted NGs and accumulation of DAD mass, along with shift in dissipation factor, was traced over 24 hours (b). Modeled NG shear modulus shifted from $135.60 \pm 25.13 \mathrm{kPa}$ to $197.03 \pm 48.52 \mathrm{kPa}$ after addition of $25.22 \% \pm$ $4.40 \%$ mass via DAD $(n=7,(b)-(c))$. 


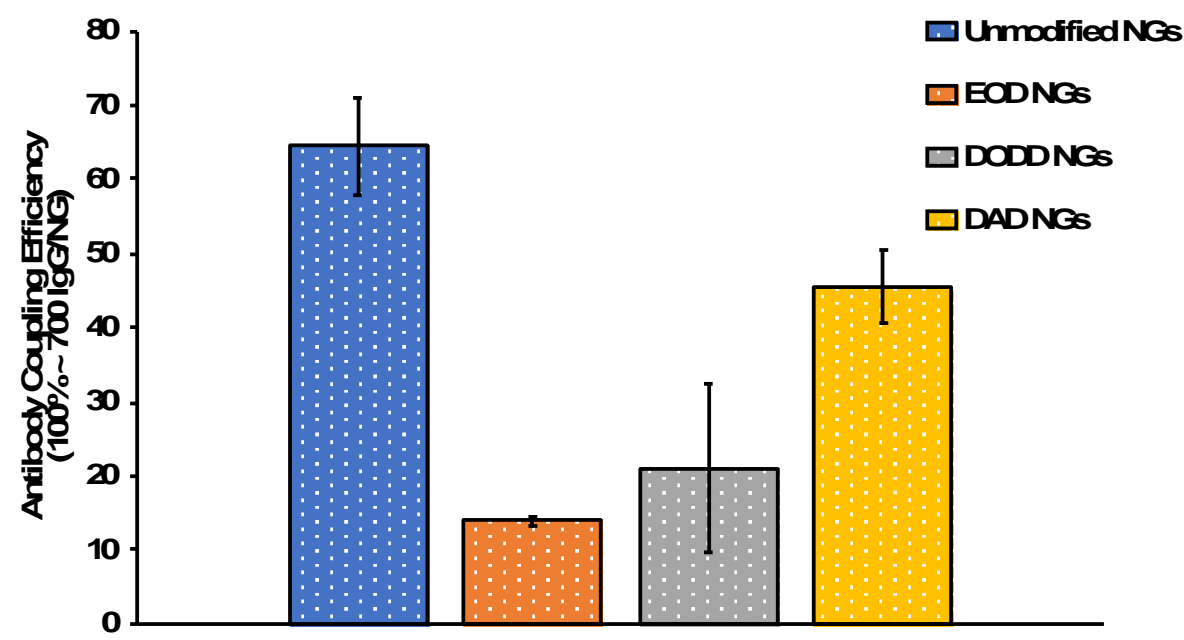

\section{Supplementary Figure 11}

Efficiency of antibody coupling to NGs before and after addition of cross-linkers. For NGs without cross-linker, antibody coupling achieved $64.49 \% \pm 6.58 \%$ efficiency, corresponding to $\sim 450$ antibodies per NG (blue bar). EOD-cross-linked NGs accumulated antibody at $13.86 \% \pm 0.61 \%$ efficiency, corresponding to $\sim 100$ antibodies per NG (orange bar). DODD-cross-linked NGs accumulated antibody at $21.05 \% \pm$ $11.40 \%$ efficiency, corresponding to 150 antibodies per NG (gray bar). DAD-crosslinked NGs accumulated antibody at $45.59 \% \pm 4.95 \%$ efficiency, corresponding to $~ 325$ antibodies per NG (yellow bar). 

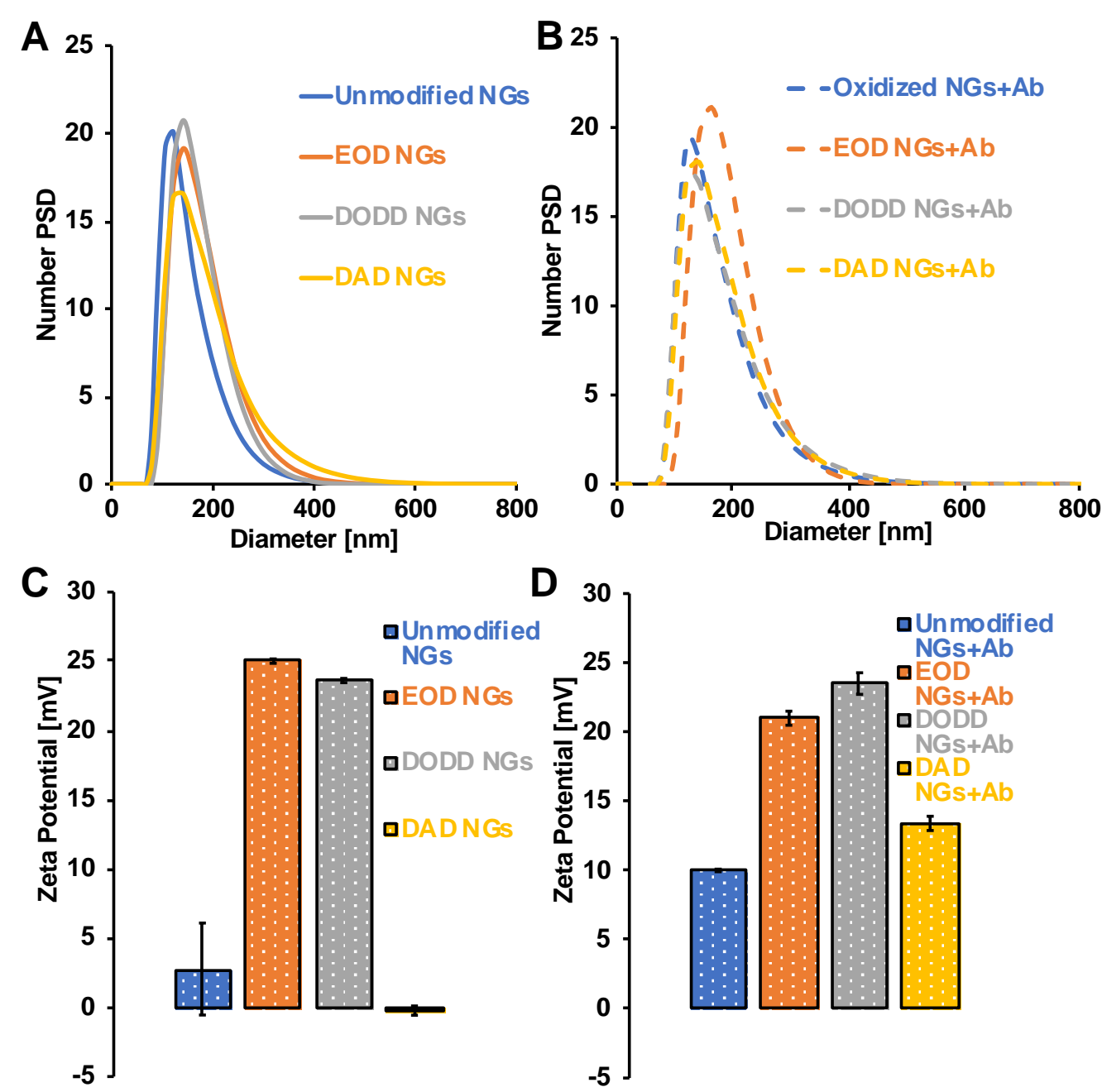

\section{Supplementary Figure 12}

DLS size distributions and zeta potential characterization of antibody-conjugated NGs. (a) reproduces data presented in Figure $2 \mathrm{~F}$ in the main text. In (b), shifts to a larger size were recorded after antibody addition for all species of NG. Distribution for unmodified $\mathrm{Ab}-\mathrm{NGs}$ reflects a diameter of $202.8 \pm 0.2 \mathrm{~nm}$ and a PDI of $0.123 \pm 0.016$. Antibodyconjugated EOD-modified NGs has a diameter $203.8 \pm 1.6 \mathrm{~nm}$, with a PDI of $0.056 \pm$ 0.025 . Antibody-conjugated DODD NGs had a diameter of $212.0 \pm 3.0 \mathrm{~nm}$ and a PDI of $0.117 \pm 0.010$. Antibody-conjugated DAD-cross-linked NGs yielded particles had a diameter of $220.4 \pm 0.9 \mathrm{~nm}$ and a PDI of $0.141 \pm 0.004$. (c) We recorded zeta potentials of $2.75 \pm 3.32 \mathrm{mV}$ for unmodified NGs, $25.000 \pm 0.153 \mathrm{mV}$ for EOD-cross-linked NGs, $23.600 \pm 0.153 \mathrm{mV}$ for DODD-cross-linked NGs, and $-0.243 \pm 0.335 \mathrm{mV}$ for DAD-crosslinked NGs. (d) Antibody addition shifted zeta potentials to $10.0 \pm 0.1 \mathrm{mV}$ for unmodified NGs, $21.0 \pm 0.5 \mathrm{mV}$ for EOD-cross-linked NGs, $23.5 \pm 0.8 \mathrm{mV}$ for DODD-cross-linked $\mathrm{NGs}$, and $13.4 \pm 0.2 \mathrm{mV}$ for DAD-cross-linked NGs. 


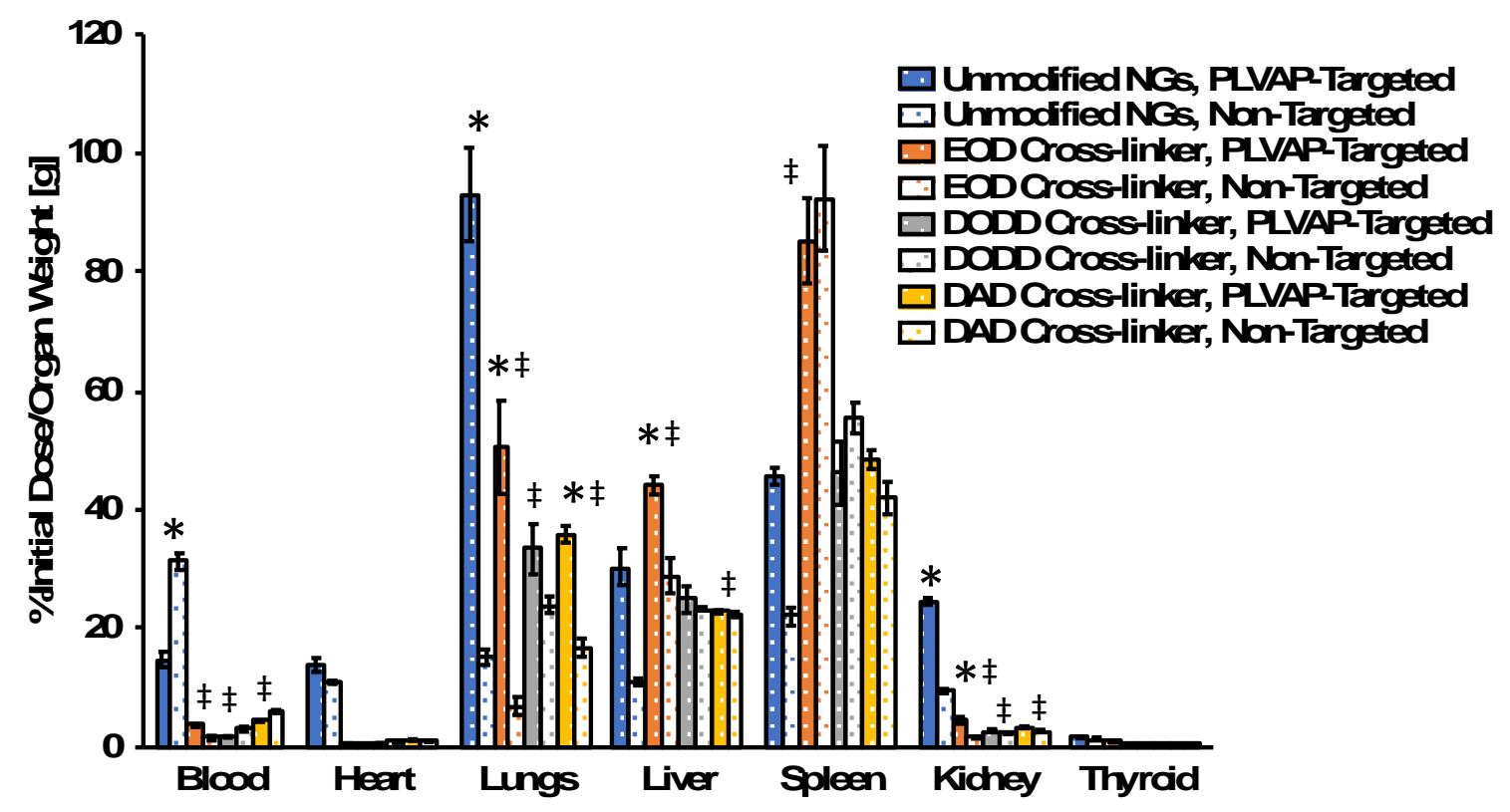

\section{Supplementary Figure 13}

Biodistributions, taken 30 minutes after intravenous bolus, for PLVAP-targeted (dark bars) and non-targeted (IgG, open bars) NGs. Yellow bars indicate DAD-cross-linked NGs, gray bars indicate DODD-cross-linked NGs, orange bars indicate EOD-crosslinked NGs, and blue bars indicate unmodified NGs. $\left({ }^{*}\right)$ indicates $p<0.01$ significance for comparison of PLVAP-targeted particles to counterpart IgG NGs in the indicated organ. A significant effect for PLVAP targeting was noted in the lungs for all particles except DODD-cross-linked NGs. Conjugation to PLVAP antibody also affected liver and kidney uptake for EOD-cross-linked NGs and limited blood pool concentration of unmodified NGs. $\left(^{\ddagger}\right)$ indicates $p<0.01$ significance for comparison of cross-linked PLVAP-targeted NGs to unmodified PLVAP-targeted NGs in the indicated organ. All species of PLVAPtargeted cross-linked NGs differed from unmodified NGs in lung uptake and blood pool concentration. 

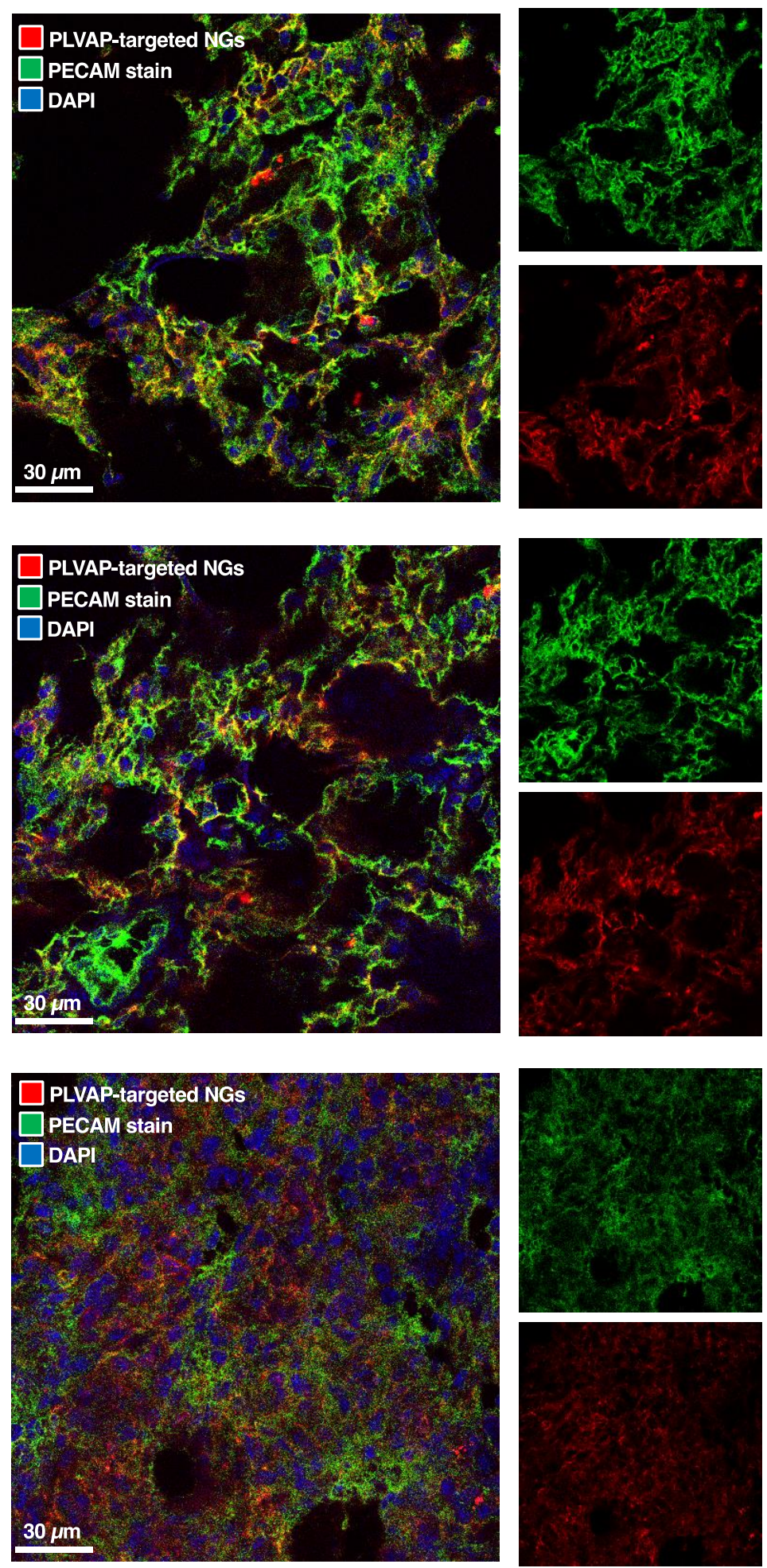

\section{Supplementary Figure 14}

Histological tracing of fluorescent PLVAP-targeted NGs in the lungs. Rhodaminebearing NGs were coated with PLVAP antibody and administered alongside fluorescent PECAM antibody. Confocal imaging of lung sections verified adhesion of PLVAPtargeted NGs in lungs with broad PECAM colocalization. 


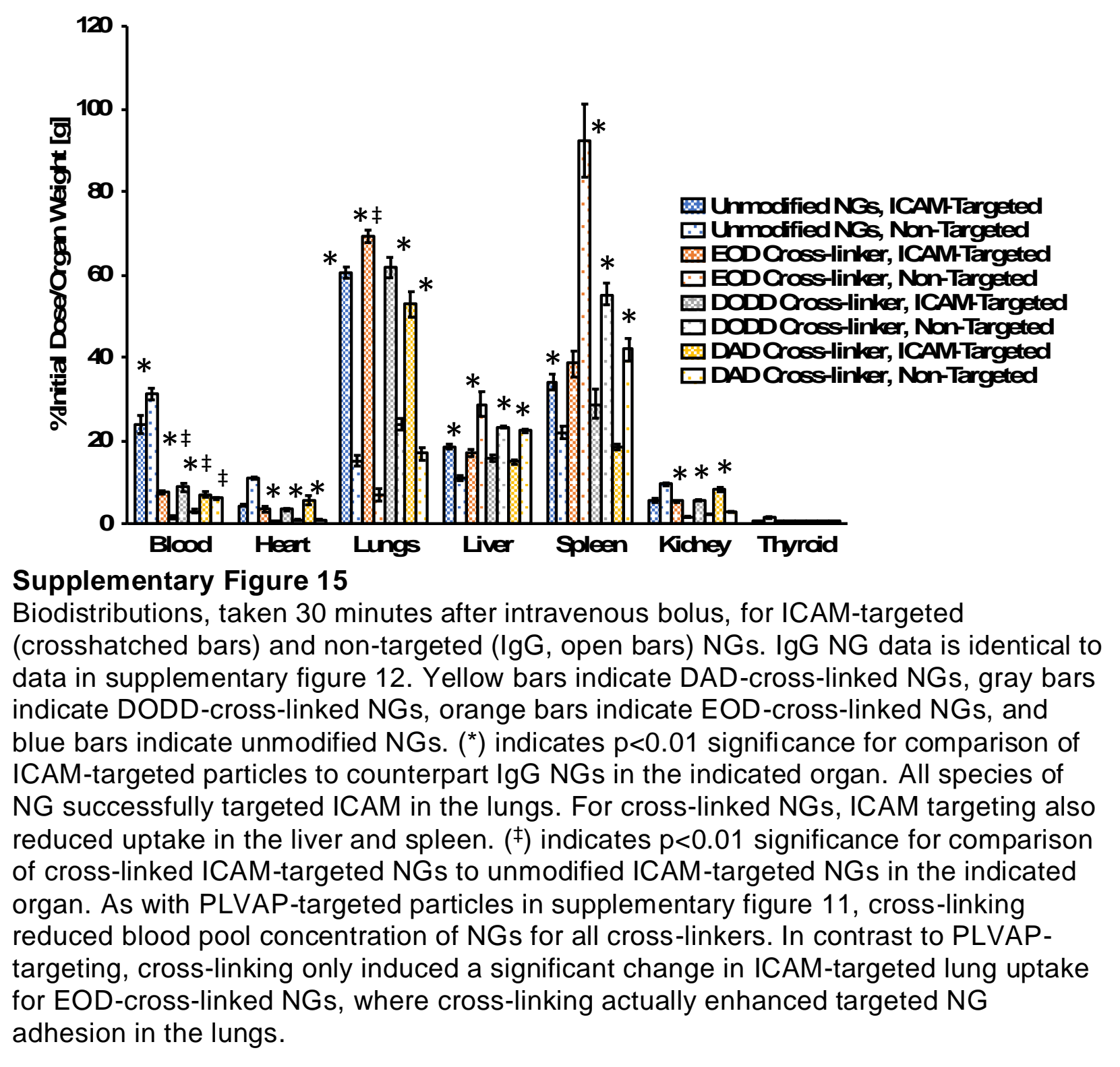




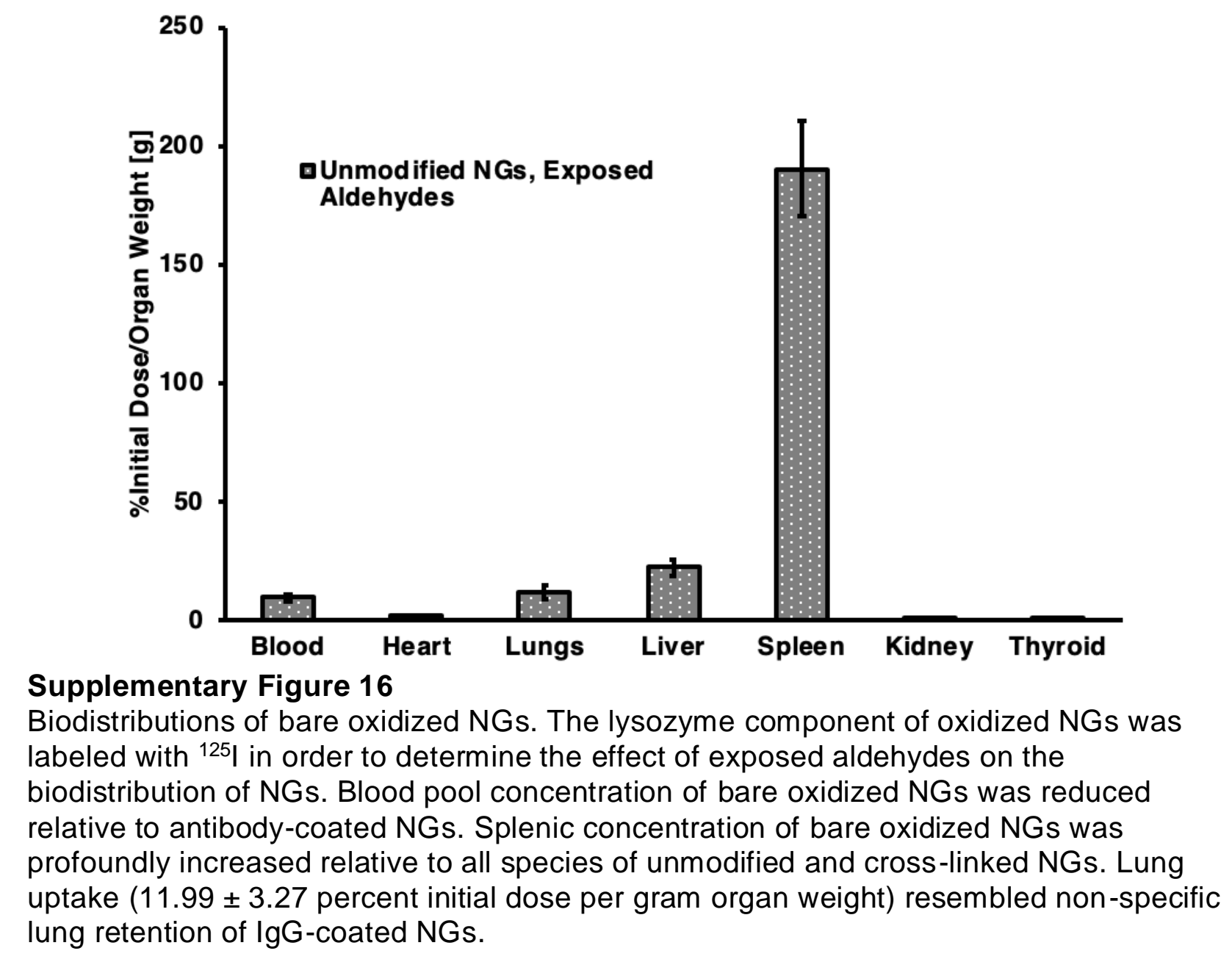




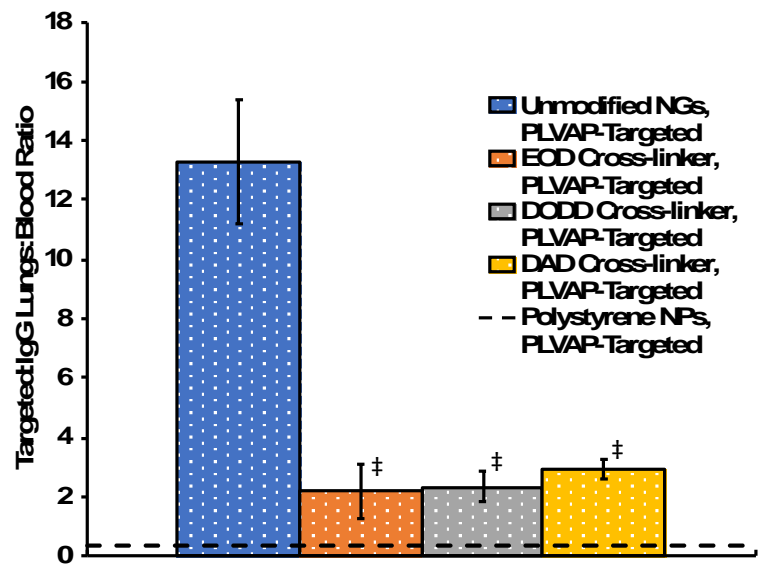

\begin{tabular}{|c|c|c|c|c|c|c|c|c|c|}
\hline \multicolumn{10}{|c|}{ Lungs:Elood Localization Ratio } \\
\hline \multicolumn{2}{|c|}{ Unmodified NGs } & \multicolumn{2}{|c|}{ EOD-linked NGs } & \multicolumn{2}{|c|}{ DODD-linked NGs } & \multicolumn{2}{|c|}{ DAD-linked NGs } & \multicolumn{2}{|c|}{ Polystyrene NPs } \\
\hline $\lg G$ & PLVAP-targeted & $\lg G$ & PLVAP-targeted & $\lg G$ & PLVAP-targeted & $\lg G$ & PLVAP-targeted & $\lg G$ & PLVAP-targeted \\
\hline $0.488 \pm 0.043$ & $6.498 \pm 0.794$ & $6.323 \pm 2.634$ & $13.732+1.692$ & $8.397 \pm 1.351$ & $19.671 \pm 4.120$ & $2.812 \pm 0.333$ & $8.215 \pm 0.249$ & $10.054 \pm 0.815$ & $5.670 \pm 1.402$ \\
\hline
\end{tabular}

\section{Supplementary Figure 17}

Clearance-normalized specificity of PLVAP-targeted NGs for mouse lungs 30 minutes after intravenous bolus. Lung uptake of NGs was normalized to blood concentration and targeting specificity was determined as the ratio of clearance-normalized parameters for PLVAP-targeted NGs and IgG NGs. Unmodified NGs outperformed all cross-linked NGs, as measured by clearance-normalized lung specificity. PLVAP-targeted crosslinked NGs did have greater clearance-normalized lung specificity than polystyrene NPs of similar size (dashed line) ${ }^{16}$. Tabular data indicates clearance-normalized lung adhesion for each type of NG. 

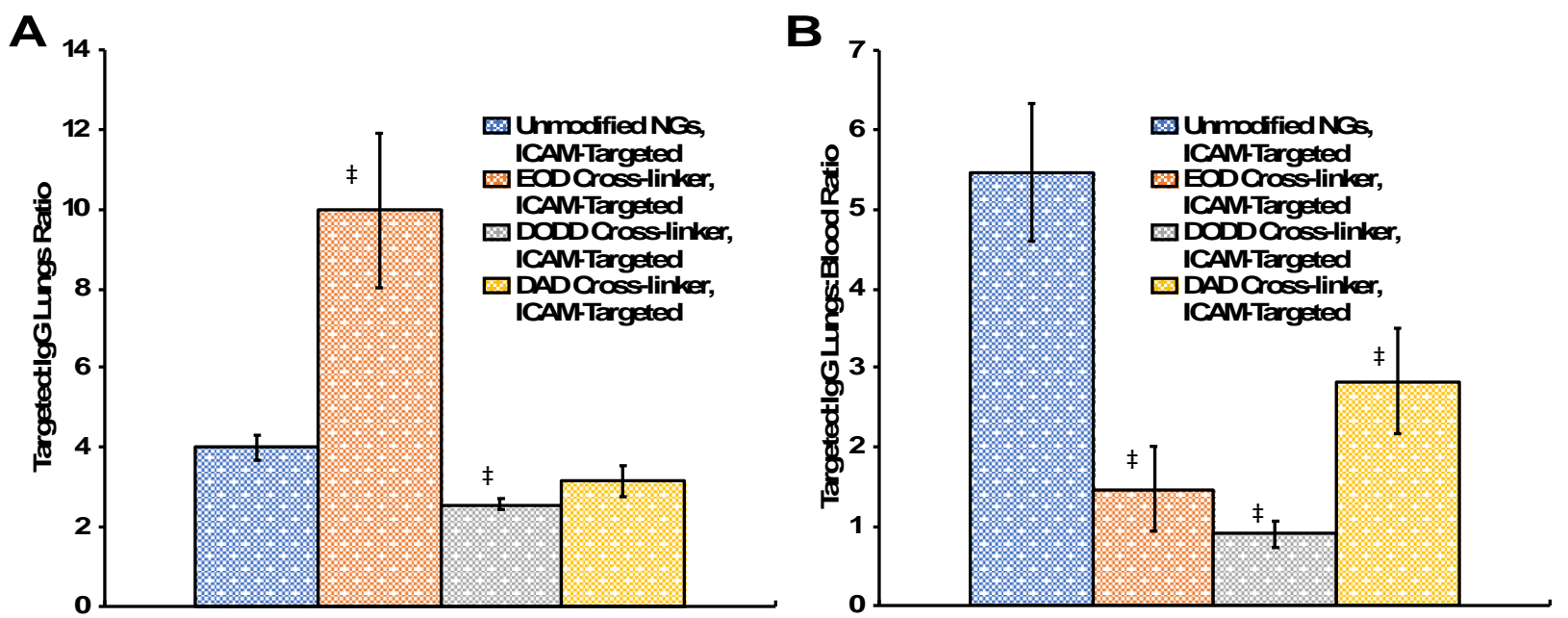

\section{Supplementary Figure 18}

Specificity of ICAM-targeted NGs for the lungs. (a) presents the ratio of ICAM-targeted lung uptake to non-targeted lung uptake for DAD-cross-linked NGs (yellow), DODDcross-linked NGs (gray), EOD-cross-linked NGs (orange), or unmodified NGs (blue). ( ${ }^{\ddagger}$ ) indicates $p<0.01$ significance for comparison of cross-linked NGs to unmodified NGs. DAD-cross-linked NGs and DODD-cross-linked NGs had lower ICAM-targeted lung specificity than more flexible EOD-cross-linked NGs or unmodified NGs. (b) presents the clearance-normalized ICAM targeting lung specificity parameter, as defined for PLVAP targeting in supplementary figure 13. By this measure, unmodified NGs, with prolonged circulation for both ICAM-targeted and non-targeted particles, outperformed all species of cross-linked particle in ICAM targeting. 


\section{Supplementary Movie Legend}

Supplementary Movie 1. Fly-through movie of confocal stack depicting a $20 \mu \mathrm{m}$ slice of mouse lungs after administration of rhodamine B PLVAP-targeted NGs (red signal in movie) and Alexa Fluor 647-conjugated PECAM antibody (green signal). Cell nuclei are highlighted by blue DAPI stain. NG signal was largely colocalized with or near to PECAM-associated signal.

Supplementary Movie 2. Movie depicting three-dimensional arrangement of PLVAPtargeted NGs and PECAM antibody in mouse lungs, as extrapolated from a confocal stack obtained as in Supplementary Movie 1. Red signal corresponds to NGs, green signal corresponds to PECAM antibody, and blue signal indicates DAPI stain for nuclei. 


\section{Gnu Octave script for analysis of AFM micrographs and modulus map data}

close all;clear all;

$\%$ Import comma-separated variable files generated in Asylum/Igor Pro software to prepare matrices of sample height and modulus over a field of view determined by "Import_Vector"

\%"Import_Force_Map" reflects analysis of modulus data and was implemented in separate runs of this script for each Hertzian modeling strategy outlined in the results and methods in the main text

Import_Vector;

Import_Force_Map;

Import_Height_Map;

$\%$ Convert axis vectors and heights to nanometers

$m x=m x^{*}\left(10^{\wedge} 9\right)$;

$m z H h=m z H h^{*}\left(10^{\wedge} 9\right)$;

$m y=m x$;

$\%$ Create modulus map

fig1=figure;

colormap('jet');

imagesc(mx,my,mz);

colorbar;

hx=xlabel('[nm]','fontsize',10)

hy=ylabel('[nm]','fontsize', 10)

$\mathrm{h}=$ colorbar () ;

set(h,"title","Modulus [Pa]");

$\%$ Set axis limit at $400 \mathrm{kPa}$

caxis([0 4e05]);

$\%$ Create height map

fig2=figure;

colormap('jet');

imagesc(mx,my,mzHh);

colorbar;

hx=xlabel('[nm]','fontsize',10)

hy=ylabel('[nm]','fontsize', 10)

$\mathrm{h}=$ colorbar();

set(h,"title","Height [nm]");

\%Prepare a mask to filter out data points in the height map below $20 \mathrm{~nm}$

ind $=$ size $(\mathrm{mz})$;

maskH=ones(ind(1), ind(2));

for $i=1$ :ind $(1)$

for $j=1$ :ind $(2)$

if $\mathrm{mzHh}(\mathrm{i}, \mathrm{j})<20$

$\operatorname{maskH}(\mathrm{i}, \mathrm{j})=0$; 


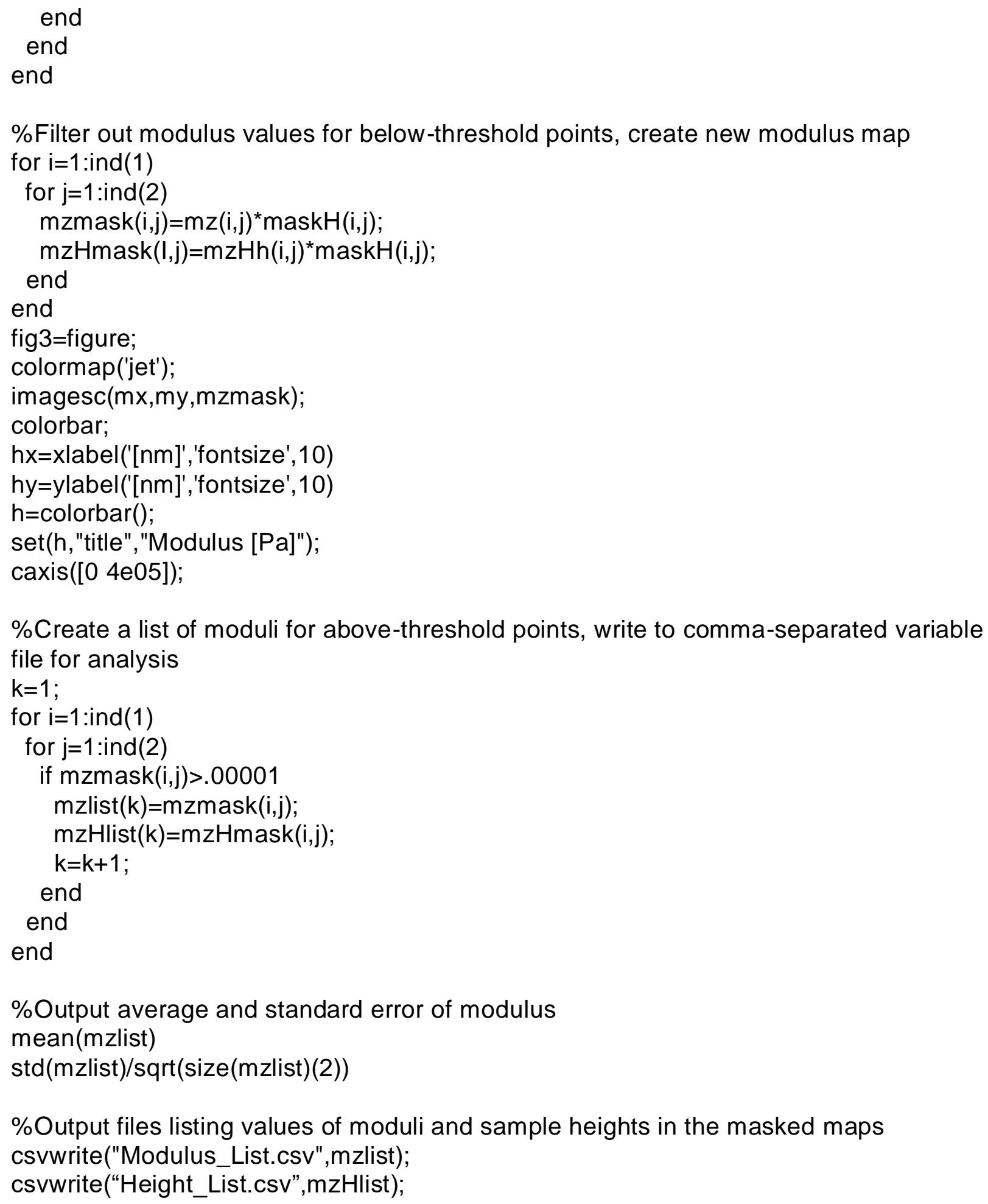

